\title{
The Effect of Turbulent Velocity Fluctuations on the Convective Heat Transfer to Droplets Subjected to Evaporation and Thermolysis
}

\author{
Ning Guo, Oskar Finnerman and Henrik Ström \\ Division of Fluid Dynamics, Department of Applied Mechanics \\ Chalmers University of Technology, SE-412 96 Gothenburg, Sweden
}

\begin{abstract}
The effect of turbulent velocity fluctuations on the convective heat transfer to single droplets in a turbulent channel flow are investigated numerically. It is found that for properties relevant to typical liquid spray applications, the convective heat transfer is enhanced with increasing droplet size and bulk Reynolds number. The combined effect of convective heat transfer enhancement and increased driving forces for heat and mass transfer due to droplet dispersion is thereafter investigated for a commercial spray application. The probability distribution functions of droplet properties in the spray are found to be significantly affected by the presence of turbulent velocity fluctuations in the carrier phase.
\end{abstract}

Keywords: turbulence, convective heat transfer, droplet flow.

PACS: 47.27.-i, 47.27.E-, 47.27.T-, 47.27.te, 47.55.D-, 47.70.Fw, 44.05.+e, 44.35.+c

\section{INTRODUCTION}

The evaporation of droplets sprayed into a turbulent gas flow is of considerable interest in a number of important industrial processes. One application of specific interest in the present work is that of a turbulent spray used as a means to deliver a liquid reducing agent to a turbulent exhaust gas. This process forms the basis for the selective catalytic and non-catalytic reduction of nitrogen oxides $\left(\mathrm{NO}_{\mathrm{x}}\right)$ using urea (urea-SCR and urea-SNCR), which are common methods to regulate the emissions of $\mathrm{NO}_{\mathrm{x}}$ from combustion processes onboard vehicles and in power plants. The primary aim is here to mix ammonia $\left(\mathrm{NH}_{3}\right)$ with the exhaust gases so that the $\mathrm{NH}_{3}$ may react efficiently with the $\mathrm{NO}_{\mathrm{x}}$. Because of safety issues, a urea-water solution is often preferred to pure $\mathrm{NH}_{3}$, as urea will form the $\mathrm{NH}_{3}$ needed when undergoing thermolysis. Consequently, the task of optimizing urea-SCR and SNCR system implies ensuring fast and efficient mixing and heating of the urea-water spray with the hot exhaust gases. For successful optimization of such systems, in-depth knowledge of the physics determining the relevant processes is therefore important.

There are several effects of turbulence on the evaporation of a droplet flow. The first effect stems from the fact that the turbulent velocity fluctuations of the carrier phase affect the convective heat transfer to the droplets. Similar effects arise from the turbulent temperature and species concentration fluctuations, which affect the driving forces for heat and mass transfer. A secondary effect from the turbulent velocity fluctuations is to disperse the droplets over a larger volume, which acts so as to increase the driving forces for heat and mass transfer to the bulk. Finally, at high droplet loadings, the turbulence characteristics of the continuous phase will be affected by the local presence of droplets.

\section{NUMERICAL METHOD}

The present work focuses on the effects of the turbulent velocity fluctuations of the carrier phase on the heat transfer to the droplets. The urea-SNCR process is used as a case study for an investigation of the combined effects of velocity fluctuations and droplet dispersion. The effects of turbulent velocity fluctuations on the predicted particle Nusselt numbers are investigated in a turbulent channel flow of varying bulk Reynolds number. The mean flow properties are taken from Kim et al. [1] and the fluctuating RMS properties are taken from Dreeben and Pope [2] (obtained at $R e=13,000$ ). As the aim of this part of the work is to assess the magnitude of the influences of the turbulent velocity fluctuations, the Reynolds-number dependence of the fluctuating quantities is disregarded. This assumption is expected to lead to an underestimation of the streamwise fluctuations and a (smaller) overestimation

International Conference of Numerical Analysis and Applied Mathematics 2015 (ICNAAM 2015)

AIP Conf. Proc. 1738, 030007-1-030007-4; doi: 10.1063/1.4951763

Published by AIP Publishing. 978-0-7354-1392-4/\$30.00 
of the wall-normal fluctuations, as shown by Wei and Willmarth [3]. The use of RMS property correlations obtained at a fixed bulk Reynolds number will thus tend to produce conservative estimates.

For each bulk Reynolds number, the complete Nusselt number history is acquired for an ensemble of 10,000 particles over a time period equal to 100 times the particle response time. The particles are introduced at random locations over the duct cross-section at the local mean velocity of the gas and are assumed to rebound in elastic collisions with the wall. The particle position is updated from knowing its velocity, which is obtained from:

$$
\frac{d u_{p}}{d t}=\frac{18 \mu}{\rho_{p} d_{p}^{2}}\left(1+0.15 \operatorname{Re}_{p}^{0.687}\right)\left(\bar{u}+u^{\prime}-u_{p}\right)
$$

Here, $u_{p}$ is the particle velocity, $\mu$ is the dynamic viscosity of the gas phase, $\rho_{p}$ is the particle density, $d_{p}$ is the particle diameter, $R e_{p}$ is the particle Reynolds number, $\bar{u}$ is the gas phase mean velocity and $u^{\prime}$ is the current gas phase velocity fluctuation (both taken at the current location of the particle). Equation (1) relates the particle acceleration to the drag force on the particle, for which the drag correlation of Schiller and Naumann is employed [4]. The time step used in the integration of these equations is two orders of magnitude smaller than the particle response time. The gas phase velocity fluctuation is updated according to an eddy-interaction model [5] as described by Dehbi [6]. The anisotropy of the velocity fluctuations in the near-wall region is accounted for by the present method, and the random insertion of particles into the duct enables the acquisition of cross-section averaged data.

The Nusselt number correlation used to assess the turbulent effects on heat transfer is taken from Frössling [7]. In a case where turbulent velocity fluctuations are not accounted for, the inserted particles would simply follow the gas flow at zero relative velocity, resulting in a constant Nusselt number equal to 2 . The Nusselt number history obtained for each particle is therefore compared to this value to determine the time-averaged increase in the Nusselt number.

The effects of turbulent velocity fluctuations are also investigated in a commercial urea-SNCR process. The injection of the urea-water solution will result in two processes - evaporation of water and thermolysis of urea [8] that can both be modeled as evaporation processes [9]. The non-isothermal, chemically reactive turbulent gas flow is modeled using the Standard k- $\varepsilon$ turbulence model and an Eulerian-Lagrangian approach is used to predict the droplet flow. The governing partial differential equations for the continuous phase are of the form displayed in equation (2), with $\Phi$ representing either mass, momentum, energy, turbulent kinetic energy or turbulent kinetic energy dissipation rate. The exchange of mass, momentum and energy with the dispersed phase are accounted for within the source terms. The governing equations for the dispersed phase are ordinary differential equations of the same form as equation (1).

$$
\frac{\partial \Phi}{\partial t}+\nabla \cdot(\Phi u)=\nabla \cdot(\Gamma \nabla \Phi)+S_{\Phi}
$$

The discretization of the continuous phase governing equations is carried out using the first-order implicit scheme for the transient terms, the second-order upwind scheme for the convective terms and the second-order central differencing scheme for the diffusion terms. The dispersed phase governing equations are discretized using a second-order implicit trapezoidal scheme. This setup has been validated for urea-SCR in our previous work [8,9] and recently also against experiments for urea-SNCR.

The effects of velocity fluctuations on the droplet trajectories and heat transfer coefficients are accounted for via the Discrete Random Walk (DRW) model [6]. Two-way coupling is employed between the gas phase and the droplet phase, so that the secondary effect of the droplet dispersion (via the temperature and concentration fields) is accounted for as well. Turbulent fluctuations of the temperature and the species concentrations and turbulence modulation from the presence of droplets are neglected, however. It is to be expected that the effect of species concentration fluctuations is negligible, whereas the effect of temperature fluctuations, if included, would act so as to further increase the broadening of the particle diameter probability distribution function [10]. The injected droplets are assumed to follow a uniform diameter distribution of either 45 or $150 \mu \mathrm{m}$. The initial urea content is $32.5 \%$ by weight, the rest is water. The droplets are injected at $300 \mathrm{~K}$ into exhaust of $1173 \mathrm{~K}$. The exhaust gases flow through a circular duct of $1 \mathrm{~m}$ diameter and $5 \mathrm{~m}$ length. The injector is located $1 \mathrm{~m}$ downstream the gas inlet and points in the co-current direction. The bulk Reynolds number is approximately 400,000 and profiles representative of fully developed turbulent flow at the current conditions are prescribed for all solution variables at the gas inlet. 


\section{RESULTS AND DISCUSSION}
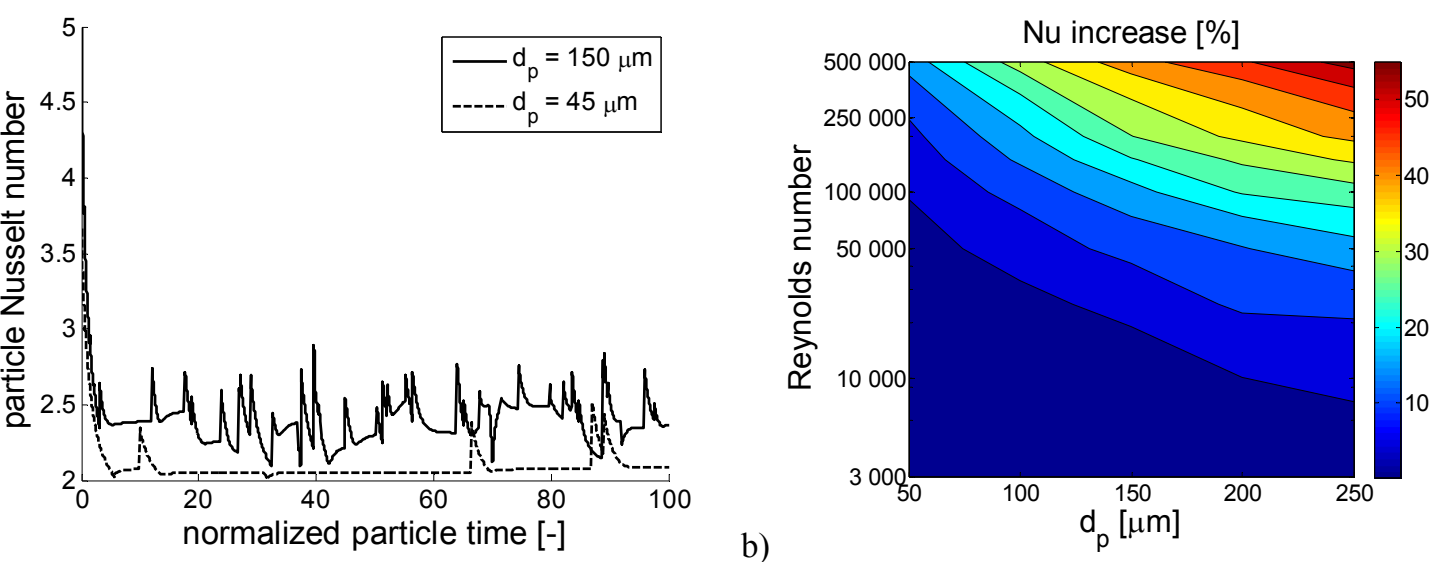

FIGURE 1. a) Illustration of the fluctuations in the particle Nusselt number as a function of normalized time for $\operatorname{Re}=130,000$.

b) Increase in the particle Nusselt number as a function of the bulk Reynolds number of the channel flow.

Typical time-histories of the particle Nusselt number in the turbulent channel flow are illustrated in Figure 1a for particles of two different diameters. The particles are inserted into the flow at the centerline between the two walls of the channel, and may thereafter move in the wall-normal direction (i.e. in a direction of increasing or decreasing RMS fluctuation magnitudes and mean streamwise velocity) as dictated by equation (1). The smaller particle has a short response time in relation to the turbulent time scale, and it interacts with only a few turbulent eddies during the investigated time span. Each time the smaller particle enters a new turbulent eddy, the relative velocity peaks, resulting in a peak also in the Nusselt number. Because of its short response time, the smaller particle is quickly accelerated by the eddy. The larger particle has more inertia, causing it to interact with a larger number of turbulent eddies during the investigated time span. The increased inertia also means that the particle will spend a significantly longer part of its life at a non-zero relative velocity to the carrier gas. It is clear from this figure that the average convective heat transfer coefficient to the droplet will be significantly affected by the turbulent velocity fluctuations.
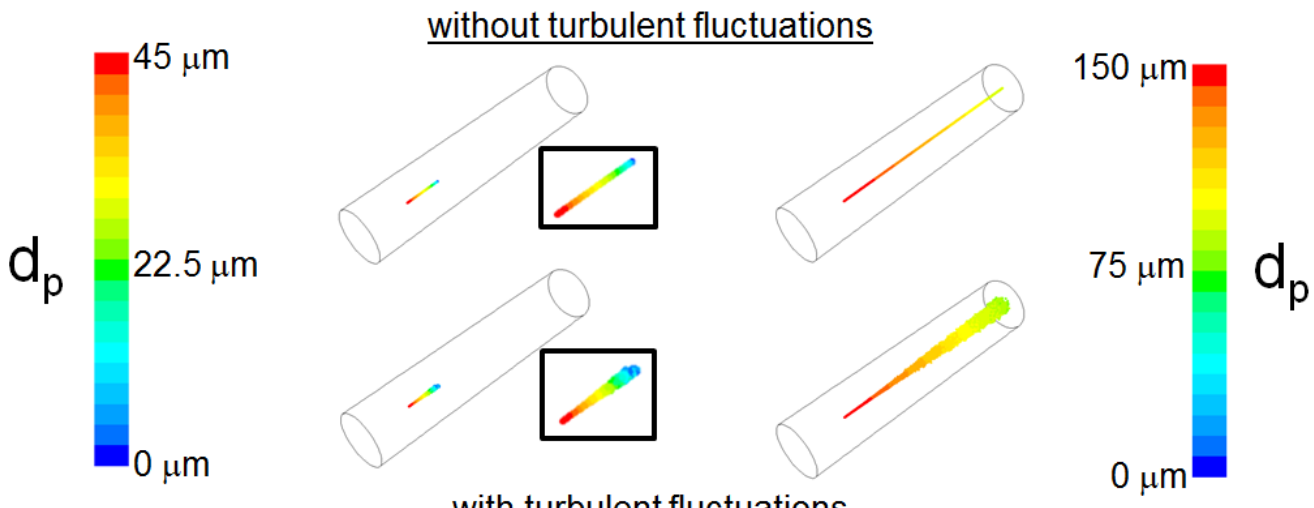

FIGURE 2. Illustrations of the predicted spray evolution for four computational urea-SNCR cases. The initial droplet size is either 45 (left) or $150 \mu \mathrm{m}$ (right) and the model for turbulent dispersion of the droplets is either turned on (bottom) or off (top).

The framed boxes show close-ups of the spray for the $45 \mu \mathrm{m}$ cases.

Figure $1 \mathrm{~b}$ shows an overview of the effects of the turbulent velocity fluctuations on the average Nusselt number. The largest increase in the Nusselt number due to velocity fluctuations of the carrier phase are observed for large particles at high bulk Reynolds numbers. The increased effect at high Reynolds numbers is likely to be most significantly affected by the fluctuations in the wall-normal direction, as these fluctuations are the only ones moving the particles in a direction of changing mean velocity. Increasing the particle diameters implies increasing the 
particle response times, and hence explains why larger particles spend a comparatively longer time in relative motion to the fluid surrounding them. The observation by Berlemont et al. [10], that the effect is most pronounced for intermediate-sized droplets (appx. $100 \mu \mathrm{m}$ ), is not valid here since the Lagrangian time scale of the turbulence of the fully developed channel flow is longer than that of the grid turbulence they used as a basis for their investigation.

Numerical simulations accounting for the effects of varying mean temperature and species concentrations in the bulk as well as the diameter changes of the evaporating droplets are performed, as shown in Figure 2. The radial dispersion of droplets that the turbulence is responsible for is clearly visible. The sizes of droplets exiting the pipe in the case of $150 \mu \mathrm{m}$ initial diameter are also smaller when turbulent velocity fluctuations are taken into account. An investigation of the droplet properties (droplet Reynolds number and temperature) is provided in Figure 3. The distribution of $R e_{p}$ highlights the existence of larger relative velocities between the droplets and the gas in the cases where turbulent velocity fluctuations are accounted for. In these cases, the distribution of droplet temperatures also shifts towards higher values for the droplets undergoing thermolysis. The thermolysis temperature is determined by a balance of heat and mass transfer effects, indicating that the convective heat transfer to the droplet also affects the thermolysis process. As the urea that is produced partakes in a set of fast and competing gas phase reactions, the thermolysis temperature is an important parameter for the prediction of the efficiency of a urea-SNCR system.
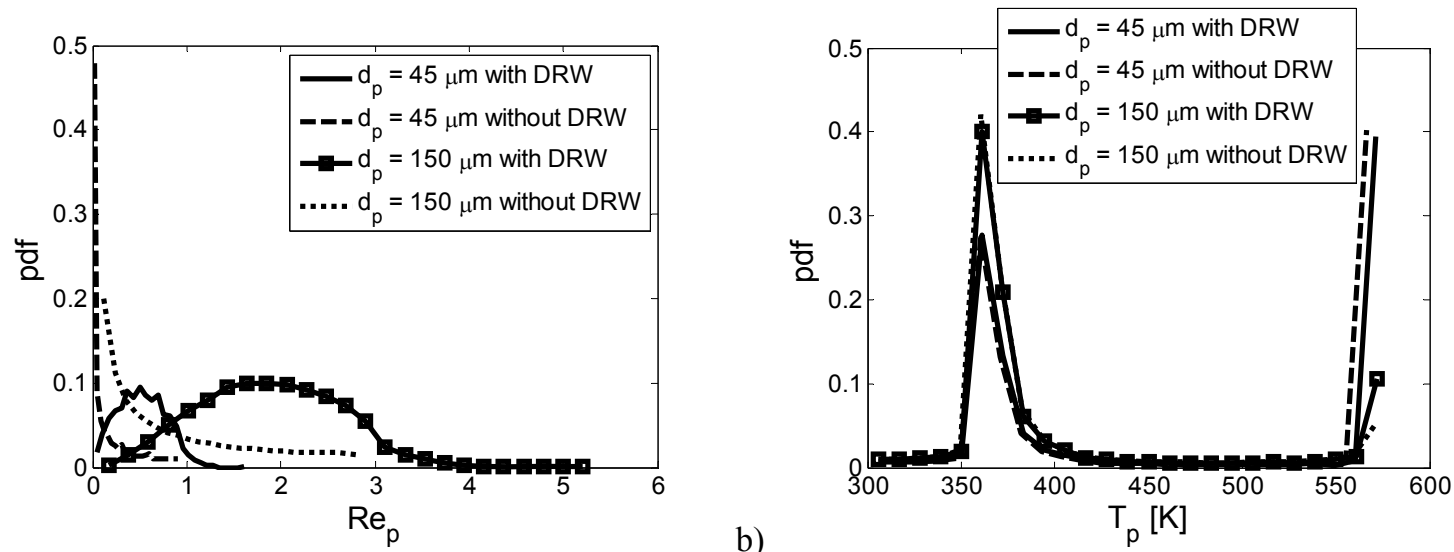

FIGURE 3. Probability distribution functions of droplet properties in the urea-SNCR simulations: a) the droplet Reynolds number, and b) the droplet temperature.

\section{CONCLUSIONS}

In the current work, the enhancement of the convective heat transfer to droplets subjected to evaporation and/or thermolysis is investigated numerically. It is shown how the increase in the heat transfer coefficient, as predicted from an eddy-interaction model, increases with the droplet size and the Reynolds number of the bulk flow. Furthermore, the interplay of primary and secondary effects of turbulent velocity fluctuations on the heat transfer to a turbulent spray is investigated for a commercial urea-SNCR application. It is found that predictions of the thermolysis temperature are sensitive to the modeling of turbulent velocity fluctuations.

\section{REFERENCES}

1. J. Kim, P. Moin and R. Moser, J. Fluid Mech. 177, 133-166 (1987).

2. T. D. Dreeben and S. B. Pope, Phys. Fluids 9, 154-163 (1997).

3. T. Wei and W. W. Willmarth, J. Fluid Mech. 204, 57-95 (1989).

4. L. Schiller and Z. Naumann, Zeitschrift des Vereines Deutscher Ingenieure 77, 318-320 (1935).

5. A. D. Gosman and E. Ioannides, J. Energy 7, 482-490 (1983).

6. A. Dehbi, Nucl. Eng. Des. 238, 707-715 (2008).

7. N. Frössling, Gerl. Beitr. Zur Geophysik 52, 170-216 (1938).

8. H. Ström, A. Lundström and B. Andersson, Chem. Eng. J. 150, 69-82 (2009).

9. A. Lundström, B. Waldheim, H. Ström and B. Westerberg, J. Automobile Eng. 225, 1392-1398 (2011).

10. A. Berlemont, M.-S. Grancher and G. Gouesbet, Int. J. Heat Mass Transfer 34, 2805-2812 (1991). 